Research Paper

\title{
Dentinal mineralization is not limited in the mineralization front but occurs along with the entire odontoblast process
}

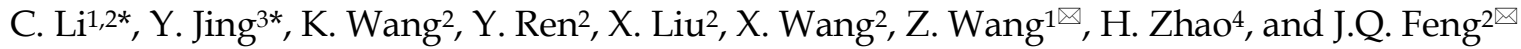 \\ 1. Shanghai Engineering Research Center of Tooth Restoration and Regeneration, Department of Oral Implant, School of Stomatology, Tongji University, \\ Shanghai 200072, PR China. \\ 2. Department of Biomedical Sciences, Texas A\&M University College of Dentistry, Dallas, TX, 75246, USA \\ 3. Department of Orthodontics, Texas A\&M University College of Dentistry, Dallas, TX, 75246, USA \\ 4. Department of Restorative Dentistry, Texas A\&M University College of Dentistry, Dallas, TX, 75246, USA \\ ${ }^{*}$ Authors equally contributed to this work.
}

$\triangle$ Corresponding authors: Jian Q. Feng: jfeng@tamhsc.edu, Department of Biomedical Sciences, Texas A\&M University College of Dentistry, Dallas, TX, 75246, USA. +1-214-370-7235 or Zuolin Wang: zuolin@tongji.edu.cn, Shanghai Engineering Research Center of Tooth Restoration and Regeneration, Department of Oral Implant, School of Stomatology, Tongii University, Shanghai 200072, PR China. +86-21-66313725

( $)$ Ivyspring International Publisher. This is an open access article distributed under the terms of the Creative Commons Attribution (CC BY-NC) license (https://creativecommons.org/licenses/by-nc/4.0/). See http://ivyspring.com/terms for full terms and conditions.

Received: 2018.02.25; Accepted: 2018.03.25; Published: 2018.04.30

\begin{abstract}
The mineralization-front theory is historically rooted in mineralization research fields for many decades. This theory is widely used to describe mineralization events in both osteogenesis and dentinogenesis. However, this model does not provide enough evidence to explain how minerals are propagated from the pulp-end dentin to dentin-enamel junction (DEJ). To address this issue, we modified the current research approaches by a) extending the mineral deposition windows of time from minutes to hours, instead of limiting the mineralization assay on days and weeks only; b) switching a regular fluorescent microscope to a more powerful confocal microscope; in which both mineral deposition rates and detail mineral labeling along with dentin tubules can be documented; and c) using reporter mice, including the Glil-CreERT2 activated tomato and the 2.3 Coll-GFP to mark odontoblast processes combined with mineral dye injections. Our key findings are: 1) Odontoblast-processes, full of numerous mini-branches, evenly spread to entire dentin matrices with a high density of processes and a large diameter of the main process at the predentin-dentin junction; and 2) The minerals deposit along with entire odontoblast-processes and form many individual mineral collars surrounding odontoblast processes. As a result, these merged collars give rise to a single labeled line at the dentin-predentin junction, in which the dental tubules are wider in diameter and denser in odontoblast processes compared to other dentin areas. We therefore propose that it is the odontoblast-process that directly contributes to mineralization, which is not simply limited in the mineralization front at the edge of dentin and predentin, but occurs along with the entire odontoblast process. These new findings will shed new light on our understanding of dentin structure and function, as well as the mechanisms of mineralization.
\end{abstract}

Key words: Mineralization front, dentinogenesis, odontoblast, cell lineage tracing, tooth development, dentin mineralization

\section{Introduction}

Bone and tooth dentin are two fundamental mineralized tissues that share many similarities, especially in the mineralization process. For decades, the mechanism of mineralization in osteogenesis and dentinogenesis has been a research focus due to its critical role in physiological and pathological processes. The theory of the mineralization front is currently described as a newly-formed, superposed 
mineral layer on either the bone edge and the osteoid layer [1-3] or the dentin edge close to predentin[4, 5]. The mineralization front theory was developed using techniques like double tetracycline labeling assays which trace previous mineralization events. The incorporation of tetracycline into bone reflects the site of mineralization at the time of the tetracycline administration. This method is also widely used for studies of bone remodeling activity, allowing the mineral deposition rate to be calculated $[6,7]$. The theory of mineralization solely occurring at the mineralization front was indirectly supported by an assay using ${ }^{33} \mathrm{P}$ and ${ }^{3} \mathrm{H}$-serine labeling phosphoproteins, which showed incorporation of these molecules into the predentin and dentin junction; this was interpreted as the mineralization front by these authors [8,9]. In addition, Lundgren et al. demonstrated significant high $\mathrm{Ca}^{2+}$ ion activity in the mineralization front using calcium-specific micro-electrode methodology [10]. However, it is well documented that dentin mineral is evenly distributed in dentin matrix with many hypermineralized collars (known as the peritubular dentin) surrounding odontoblast processes, indicating a likely mechanism by which mineralization occurs through odontoblast processes. Furthermore, a sulfated component (chondroitin sulfate) was recently identified within the hypermineralized collars of the organic matrix of bovine peritubular dentin [11]. These authors speculate that the presence of sulfur co-localized with calcium may sequester calcium ions allowing for the entire complex to later become mineralized. Thus, there may be an alternate process: mineralization occurs throughout the odontoblast process, instead of limited in the edge of dentin and predentin.

Although the branching and extent of odontoblast processes in dentin matrices have been reported on many times, a few studies raised different views on odontoblast process structure. For example, the early electron transmission work showed that odontoblast-processes were located in the inner dentin layer, and never extended more than half distance between the pulp and the dentin-enamel joint (DEJ) [12]. The acid-etched and non-etched SEM studies in bovine dentin tissues showed an ironical finding: the diameter of dentin tubules is more than two-fold bigger in the outer region than that in the inner region (i.e., adjacent to pulp) [13]. A recent work by Khatibi et al. suggested that mini-branches of odontoblast processes exist only in the start and end terminals of the odontoblast process [14]. Thus, there is a need to re-investigate the distribution of odontoblast-processes in dentin tissues, which will not only solve the above debate but also help to understand mineralization events.

\section{Materials and Methods}

\section{Mice}

2.3 Col1-GFP[15] (2.3Col1), Gli1-CreER ${ }^{T 2}$ (Gli1) [16], Rosa $26^{\text {tdTomato }}[16]$, and Dmp $1-/$ - mice $[17,18]$ were bred and maintained at Texas A\&M College of Dentistry. 2.3Col1 and Gli1 reporter lines were generated to define the relationship between odontoblast processes and mineralization; Dmp1 -/was utilized as an established mineralization defect model. Gli1 mice were activated with a one-time intraperitoneal (IP) injection of tamoxifen, $1.5 \mathrm{mg} / 10 \mathrm{~g}$, at postnatal day 3 (T5648; Sigma-Aldrich, St. Louis, MO, USA). For better visualization of odontoblast processes, two-week-old Dmp1 -/- mice were used in this study. Four mice of each age, male and female, ages P5 to P56 were studied. All protocols were reviewed and approved by the Institutional Animal Care and Use Committee at Texas A\&M College of Dentistry. Human tooth was collected from a 43-year-old female cadaver.

\section{Mineralization Labelling}

To study mineralization, calcein $(5 \mathrm{mg} / \mathrm{kg})$, (190167, MP Biomedical) and alizarin-red complexone dihydrate $(20 \mathrm{mg} / \mathrm{kg}), \quad(\mathrm{A} 3882, \quad$ Sigma) were administered by IP injection. The process is based on the previously published protocol $[19,20]$. In short, a dilution solution of $0.9 \% \mathrm{NaCl}$ and $2 \% \mathrm{NaHCO}_{3}$ was used to make $20 \mathrm{mg} / \mathrm{ml}$ alizarin-red and $10 \mathrm{mg} / \mathrm{ml}$ calcein. The dye solution's $\mathrm{pH}$ was adjusted to 7.4.

To address various mineralization events, five combinations of calcein and alizarin red were administered: 1) calcein 7 days before sacrifice, alizarin red on 2 days before sacrifice, sacrificed on day $0 ; 2$ ) calcein on 20 hours before sacrifice, alizarin red 4 hours before sacrifice, sacrificed at day $0 ; 3$ ) calcein 4 hours before sacrifice, sacrificed at day 0 (tamoxifen- induced Gli1 mice); 4) alizarin red 4 hours before sacrifice, sacrificed at day 0 (2.3Col1 mice); and 5) alizarin red administered at 5, 15, 30 or 60 minutes before sacrifice, sacrificed on P5 (2.3Col1 mice).

Nondecalcified samples under age of 10-day old were fixed in $4 \%$ paraformaldehyde (PFA) then embedded in optimum cutting temperature compound (O.C.T. compound, Tissue-Tek). Samples were cryosectioned using stick transparent film (Section-Lab Co. Ltd., Hiroshima, Japan) at the thickness of $10 \mu \mathrm{m}$. Film-stick sections were mounted with DAPI and imaged with confocal microscopy. Nondecalcified samples at age of 14-day old were were dehydrated through a graded series of ethanol $(70-100 \%)$ and embedded in MMA without decalcification. Fifty $\mu \mathrm{m}$ sections were cut using a Leitz 1600 saw microtome, followed polishing by 
different sizes of sand papers to the thickness of 20 $\mu \mathrm{m}$ for confocal imaging.

\section{FITC ([2, 5]- Fluorescein isothiocyanate) staining, embedding, and sectioning}

FITC, a small fluorescent dye, was used to provide a visual representation of the organization of the odontoblasts and their processes under the confocal microscope. The detailed process was previously described [21]. Briefly, human and mouse teeth were fixed in $70 \%$ ethanol or $4 \%$ PFA for two days, followed by series of dehydration using $90 \%$, $95 \%$ and $100 \%$ of ethanol. Then, samples were stained with 1\% FITC (Sigma, cat. no. F7250) in 100\% ethanol overnight, followed by the methylmethacrylate embedding process described previously [22]. A cross section (300-400 $\mu \mathrm{m}$ thick) of tooth samples was cut with a diamond-bladed saw (Buehler, Lake Bluff, IL). Sections were then sanded and ground to a final thickness of 30-50 $\mu \mathrm{m}$ with a well-polished surface for confocal imaging.

\section{Acid-etched SEM}

For better exposure of deep odontoblast processes, we trimmed mandibular incisors of one-month-old wild type mice three-times a week for one month. The frequent tooth trim should greatly reduce the dentin mineral content, as the mineral replacement rate is slower than that of dentin matrix protein productions [23]. This technique will allow the dentin tubules to be fully exposed by the acid etching method. Methylmethacrylate embedded samples were polished as previously described [24]. Each sample was placed in an ultrasonic bath for cleaning, followed by air drying. The surfaces were etched with 37\% phosphoric acid (Acros, \#201145000) for 8 seconds, followed by $5 \%$ sodium hypochlorite (LACHAT, \#52007) for 20 minutes. The samples were then sputter coated with gold as described previously $[25,26]$, and examined using a JEOL JSM-6010 scanning electron microscope.

\section{Immunohistochemistry}

The dissected samples were fixed in 4\% PFA overnight and decalcified by $10 \%$ EDTA ( $\mathrm{pH} 7.4)$ at $4^{\circ} \mathrm{C}$, followed by $30 \%$ sucrose dehydration and cryosection with optimum cutting temperature compound (O.C.T. compound, Tissue-Tek) at the thickness of $10 \mu \mathrm{m}$. Sections were stained by: mouse anti-nestin monoclone antibody (1:100, EMD Millipore, CA, USA), and mouse anti-biglycan monoclone antibody LF-159 (1:1000, Kerafast, MA, USA). The immunofluorescent signals were detected with corresponding Alexa second antibody
(Thermofisher, 1:200) at room temperature for 2 hours.

\section{Confocal Microscope}

Fluorescent cell images were captured by SP5 Leica confocal microscope. All images were captured at wavelength ranging from $488 \mathrm{~nm}$ (green) to 561 $\mathrm{nm}$ (red) [27]. Multiple stacked images were taken at $200 \mathrm{~Hz}$ in the dimension of $1024 * 1024$.

\section{Statistical Analysis}

Statistical analysis was performed with IBM SPSS 13.0 All data were assessed by one-way ANOVA and Least Significant Difference (LSD) test. Statistical significance was considered at $\mathrm{P}<0.05 ; \mathrm{P}<0.01$; $\mathrm{P}<0.001$. Graphs were illuminated by Prism 6 (GraphPad).

\section{Results and Discussion}

\section{The entire dentinal tubule is full of numerous mini-branches with significantly higher dentinal tubules in the edge of the dentin-pulp}

To visualize the complexity of dentinal tubule structure in dentin, a 3-week-old mouse incisor specimen was stained with FITC for 24 hours. All unmineralized tissue including pulp, odontoblasts and their processes were labeled green. The confocal mages indicated FITC labeled side-branches throughout dentin tubules, from the odontoblast cell body side to the enamel-dentin junction (Fig 1a). Similar patterns of dentinal tubules and their branches were also observed in adult human dentin (Fig 1b). To detail the structure of dentin tubules further, acid-etching SEM imaging technique was employed. The 3-D SEM image data was consistent with the FITC results: dentin tubules are full of numerous mini-branches (Fig 2a). These mini-branches not only fill entire dentin matrices but connect adjacent dentin tubules (Figs 1-2). Importantly, the dentin tubule density at the pulp-end dentin is two-fold higher than that in the outer dentin area (Fig 2b), which will help to explain in part why mineral labels are strong in the "mineralization front" (see below for detail).

Together, both FITC and acid-etched SEM imaging results demonstrate that dentin tubules are composed of numerous mini-branches throughout of the entire dentin tubules; and the density of dentin tubules adjacent to the tooth-pulp edge is much higher than that in the outer dentin matrix region near the enamel-dentin junction. This unique structure feature is likely essential for the mineralization process. 

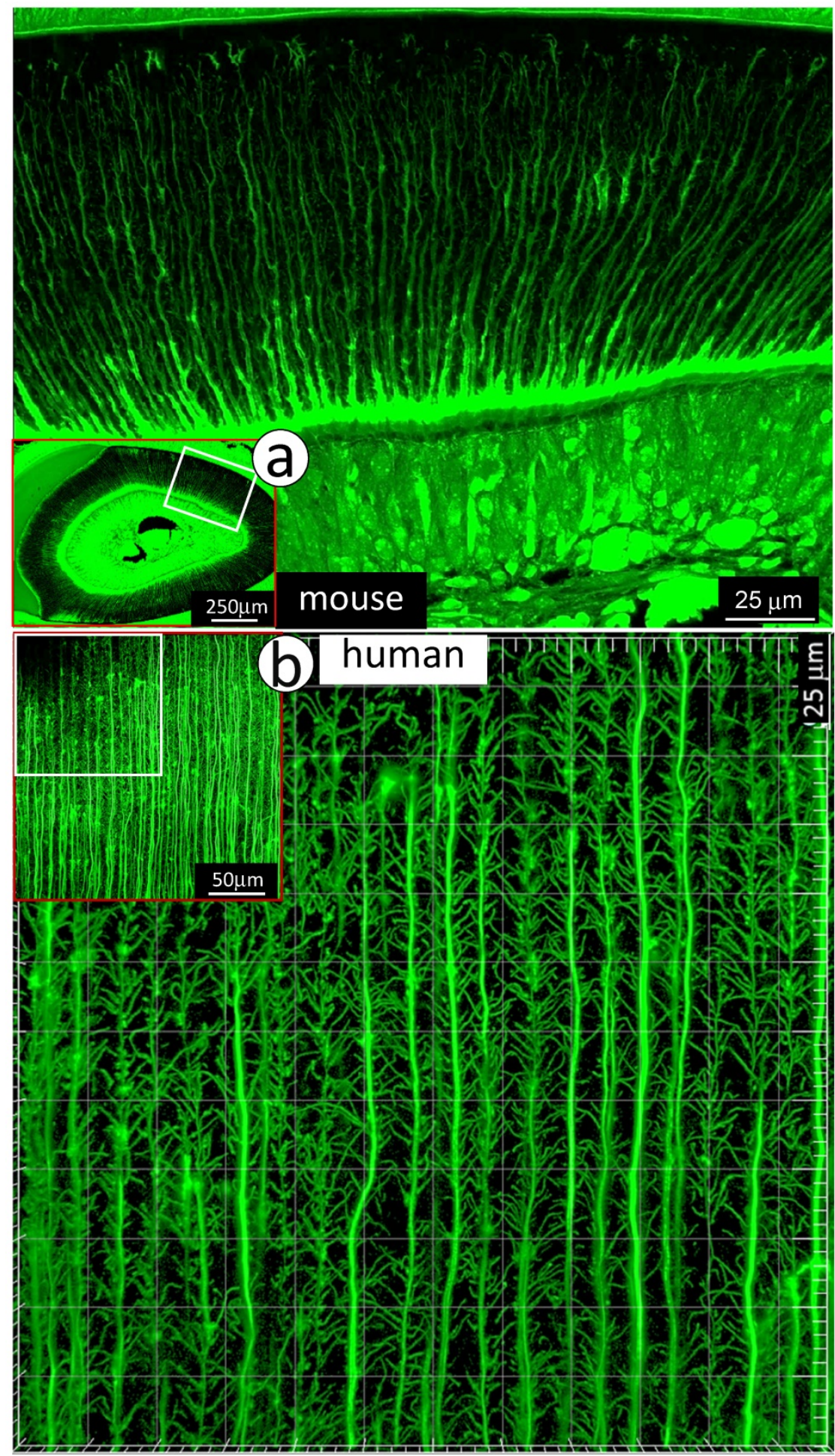

Figure 1. Distributions of mini-branches along with the entire odontoblast processes. a. The FITC confocal photograph image obtained from a 3-week-old mouse incisor, exhibiting numerous mini-dentin branches throughout the entire dentin layer with a large diameter and a high density at the edge close to the predentin layer; and $\mathbf{b}$. The 43-year-old 1st molar dentin displayed an identical distribution pattern of mini-branches along odontoblast-processes as that in the mouse incisor.

\section{Mineralization is not limited to the mineralization front but occurs throughout dentin matrices surrounding odontoblast processes.}

Because the existing theory of the mineralization front cannot explain the migration of mineral to outer dentin matrices from the pulp-end, we propose a new hypothesis based on the FITC and acid-etch SEM results presented above: dentin mineralization is odontoblast-processes dependent and occurs throughout the entire dentin matrix surrounding odontoblast-processes. To test this hypothesis, we first performed the double labeling assay in young mice with calcein injected at 
postnatal day 28 (P28), alizarin red at P33 (5 days apart), and harvested at P35. The confocal images displayed not only the expected two strong double labeled lines, the green line on the distal side and the red line adjacent to the dentin-predentin edge, but also many thin lines along with the dentin tubules (Fig 3a, and Supplementary Fig 1).
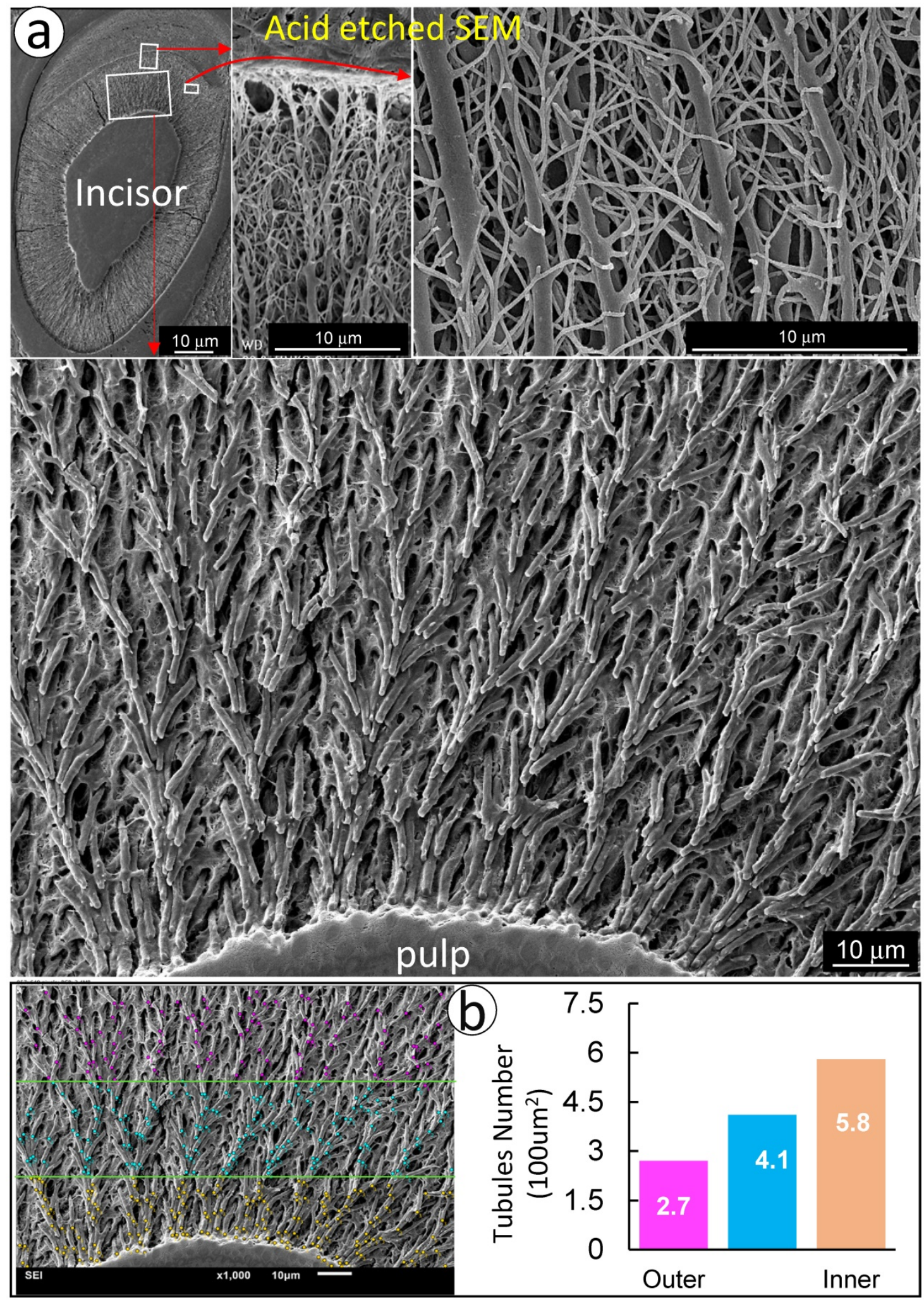

Figure 2. There are more dentinal tubules at the edge of the dentin layer adjacent to the pulp. a. The acid etched SEM image obtained from an 8-week-old incisor with frequent trim for a month displayed numerous dentin branches from the area adjacent to pulp (lower panel), middle (upper right panel) to the area adjacent to enamel (upper middle); and b. The quantitation of dentin tubular density revealed a "gradient" of dentin tubular numbers with a high density close to tooth pulp [32] and a low density toward to the enamel dentin joint area. 


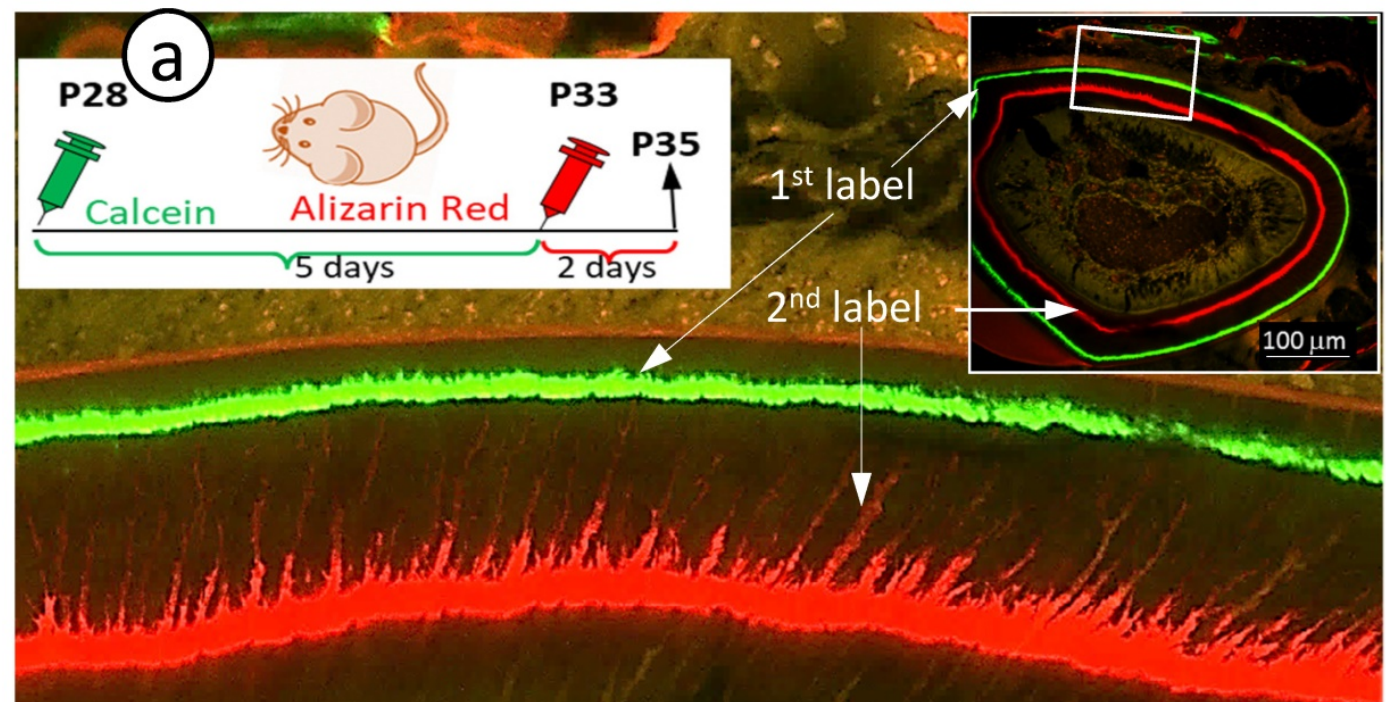

$25 \mu \mathrm{m}$

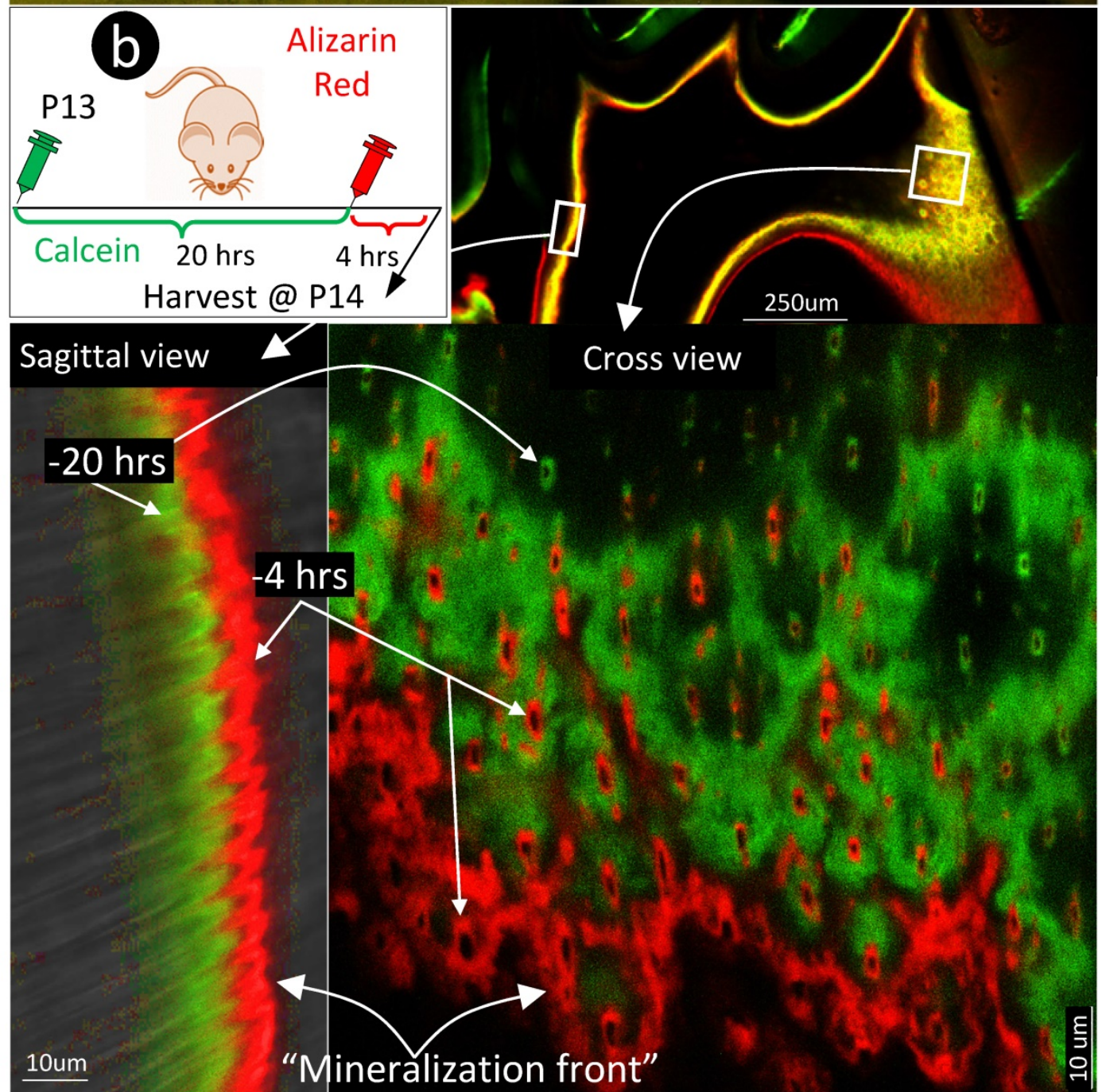

Figure 3. Mineralization occurs along with odontoblast-processes. a. A schematic illustration of long interval injections of Calcein and Alizarin-red at -7 days and -2 days respectively (top left panel); the low magnification view of the cross section of incisor (top right panel) and the enlarged confocal view of numerous labeled odontoblast-processes with the green line on the top and red line at the bottom; and $\mathbf{b}$. The confocal images of the 1 st molar labeled with Calcein at -20 hours and Alizarin Red at -4 hours revealed a clear pattern of the mineral deposited along odontoblast-processes by the enlarged sagittal view (lower left panel), and the cross view (lower right panel). Of note, the only difference of the labelled mineral collars between the surface and the inside dentin is the diameter and density of odontoblast processes. As a result of the merged mineral collars on the surface, the mineralization front is observed. 
Traditionally, the double labeling assay takes 5 to 7 days in rodents and a few weeks in large mammalians. This timeframe will not reflect the mineralization event occurred along with small dental tubules as the late added mineral will seal the mineral labeled before. Thus, we shortened the injection duration of calcein to 24 hours and alizarin red to 4 hours (20 hours apart) in the 2-week-old mice. As shown in Fig $3 b$, the short-term labeling images precisely revealed a close link of the labeled mineral and odontoblast-processes by both sagittal (left) and cross (right) views. Particularly, the cross view provided a clear mineral deposition pattern of the labelled collars in the intertubular area on both the pulp-end dentin (i.e., "mineralization front") and outer dentin. The only difference between the pulp-end dentin and outer dentin is the size and distribution of the labelled mineralized collars: wide and merged on the pulp-end and small and separated in the outer dentin, resulting in formation of the mineralization front. Because of the importance of this issue, we confirmed this finding on two more two week-old and three one-week old pups (Supplementary Fig 2), which is highly reproducible.

To enhance visualization of the dentin structures "buried" in the labelled mineral, we used Dmp1 null dentin tissues (in which mineral content is low) [28, 29] for FITC-confocal imaging. As predicted, the Dmp1-null predentin is greatly expanded, reflecting a defect in mineralization by both light and confocal microscopic images (Supplementary Fig 3), and contains abundant branches of odontoblast processes in the pulp-end dentin. Of note, many of these branches are yellow in color due to the merging of red mineral labeling and the green color of FITC, supporting the notion that mineralization occurs along with odontoblast processes. In further, we did double labeling assays (5 days apart) on control and Dmp1 null (KO) mice (Fig 4a), in which there is not only a great reduction in the mineralization rate (the commonly used readout) but also a sharp decrease in mineral labeling in both the mineralization front and inside dentin matrices (Fig 4b, right panels). These labelled-minerals appear along with dentin tubules, in which they are sparse and thin in the Dmp1 null dentin tubules compared to the age-matched control. This information further strengthens the current hypothesis that dentin tubules are essential for dentin mineralization, which is not simply limited in the mineralization front.

For better revealing the relationship between odontoblast processes and the mineralization event, we labeled 7-day-old $2.3 \mathrm{Col} 1$ (green color) mice with alizarin red for 4 hours, and 7-day-old Gli1 activated tdTomato mice (red color) with calcein (green) for 4 hours, respectively. Images precisely displayed that all odontoblast-processes are wrapped with a newly deposited mineral layer with calcein stain shown in Fig 5a \& Supplementary Fig 3 (in which dentin tubules are red); and alizarin red stain in Fig $5 b$ \& Supplementary Fig 4 (in which dentin tubules are green). In addition, these images documented two critical points: 1) "the main trunk" of the odontoblast process gradually reduces its diameter when it penetrates in outer dentin matrix; and 2) there are many branches along with the main process that directly contribute to the dentin mineralization.

Finally, we further reduced mineral labeling duration to 5, 15, 30, and 60 minutes in 5 day-old 2.3 Col 1 pups (one-time alizarin red i.p. injection), respectively. A weak mineralization sign was observed at the duration of 15-minute, which was more strong at both durations of 30- and 60-minute (Fig 6). Most importantly, the mineral deposition occurs throughout the entire dentin along with odontoblast-processes.

Of note, the current mineral labelling assay is for days and weeks to let dye "flushed out" of the cells in order to make sure that dyes are only deposited to true mineral structures. When calcein and/or alizarin red is given for short periods of time, such as minutes and hours, how do we know that the dye is truly deposited to dentin and not just flushing through the cells and processes? Firstly, none of pulp cells or odontoblast bodies or any soft tissues was stained with dyes, including predentin matrices. Secondly, the tissue process went through multiple steps with fixation solutions and dehydration processes, the non-bond dye would be "flushed out" if not bound to mineral dentin.

Collectively, we for the first time demonstrate that mineralization occurs along with the entire dentin tubules by using multiple imaging techniques in different reporter lines for labeling odontoblast processes combined with different mineral labeling dyes in both long and short windows of time points. Our data suggest that the following two factors are likely responsible for the phenomenon of the mineralization front: the large diameter of the odontoblast processes and a high process density, leading to the merge of the labeled minerals. On the other hand, in the outer dentin, the labeled mineral collars are small in diameter and far apart in distance. As a result, it is very difficult to observe these mineralization events by a regular fluorescent microscope.

This new hypothesis that mineralization occurs along with the entire odontoblast process precisely explains how and why dentin minerals are evenly distributed throughout dentin. 


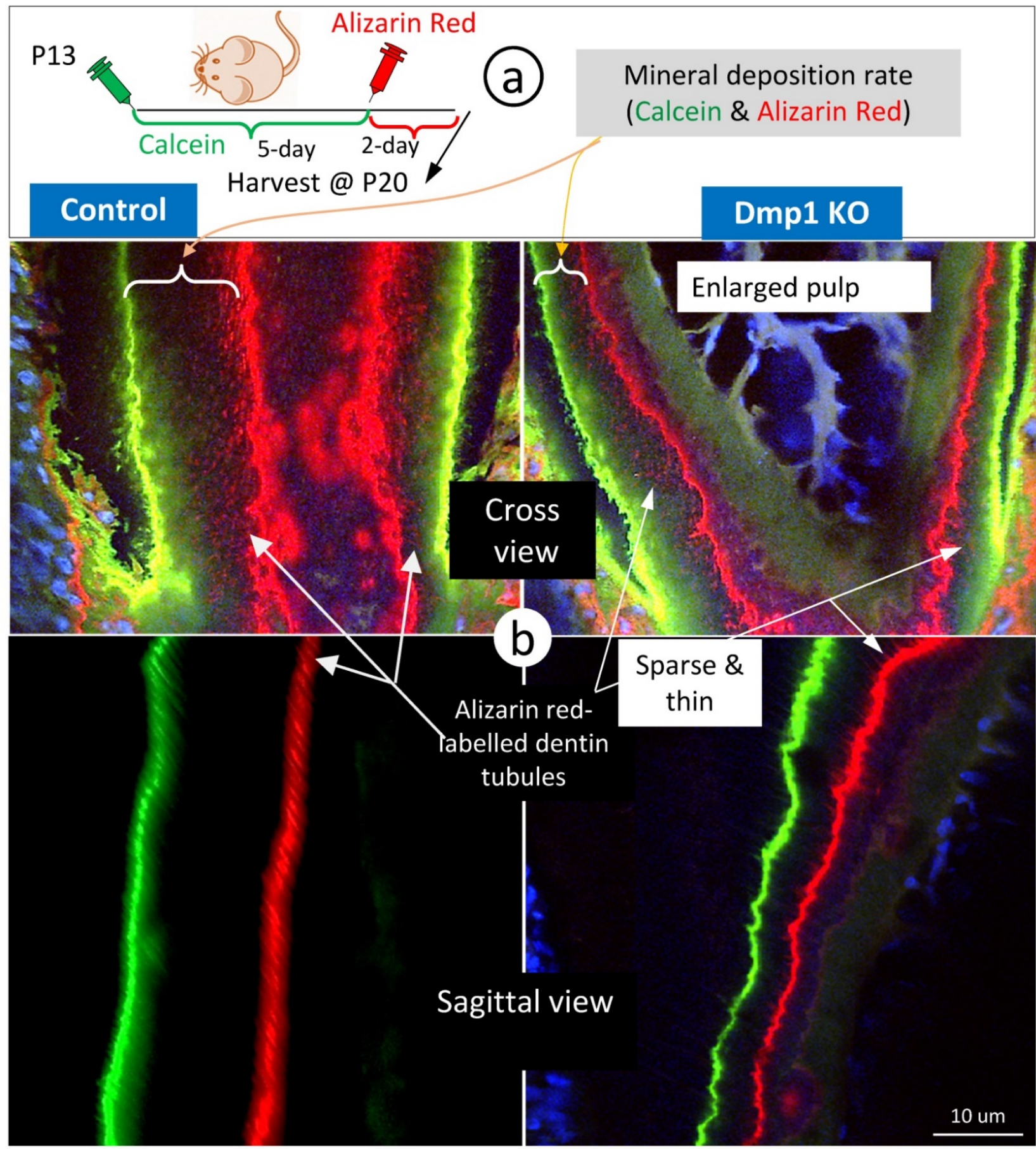

Figure 4. The mineralization rate in the Dmpl null (KO) dentin is sharply reduced in both the mineralization front and inside dentin matrices surrounding dentin tubules. a. P13 pulps (WT and Dmpl KO) were labelled with calcein and alizarin red 5 days apart and harvested for confocal imaging mineralization status; and $\mathbf{b}$. The cross (upper panels) and sagittal (lower panels) views in the Dmpl KO dentin (right panels) demonstrated 1) a great reduction in the mineralization rate; and 2) thin and sparse mineral labeling in both the mineralization front and inside dentin matrices.

In fact, this theory is consistent with the matrix vesicle theory, which is widely used to explain the initiation of mineralization during dentinogenesis [30, 31]. Based on this theory, the matrix vesicle, a small membrane-bound structure, is released into dentin matrices to provide the required molecules such as alkaline phosphatase, calcium-adenosine triphosphatase, proteoglycans, and anionic phospholipids.
These molecules bind calcium and phosphate, resulting in formation of calcium-phosphate phospholipid complexes [31]. We speculate that there are numerous matrix vesicles on the edge of dentin and predentin due to rich distributions of odontoblast-processes, which will be likely labelled with calcein or alizarin red. Furthermore, the early work using ${ }^{33} \mathrm{P}$ and ${ }^{3} \mathrm{H}$-serine co-labeling 
phosphoproteins clearly showed incorporation of junction $[8,9]$.

these molecules into the predentin and dentin

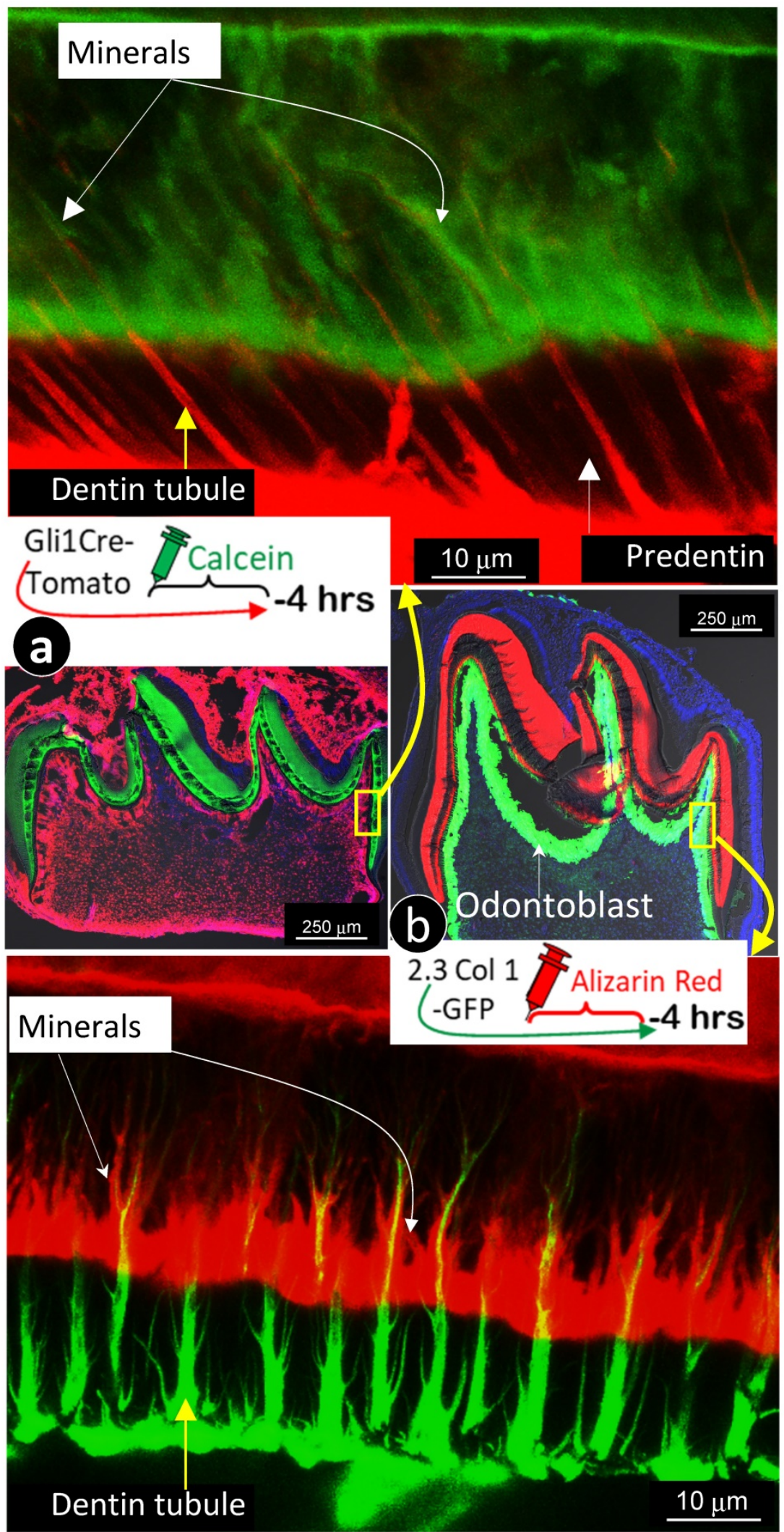

Figure 5. The labeled mineral layer is directly distributed outside of the Gli 1-tomato or the 2.3 Coll-GFP marked odontoblast processes. a. The red dentin tubules reflect the color of the Glil-Cre activated tomato expression in odontoblast processes; and the 4 hour-calcein labeled mineral matrix distributes to throughout dentin areas with a close link to all odontoblast-processes.; and $\mathbf{b}$. The distribution pattern of green dentin tubules with red mineral collars is identical to that in a. Data support the notion that mineralization takes place along with entire odontoblast processes. 


\section{Expressions of biglycan in predentin matrices and nestin in predentin tubules are correlated with a lack of mineralization in predentin.}

Next, we used immunohistochemistry stain of biglycan in the background of Gli1-activated Td-tomato mice to address the absence of a mineralization event in predentin area, where there are main odontoblast processes and numerous mini-odontoblast processes bunches (Fig 5a and Supplementary Fig 4). The rationale for selection of biglycan is that both Dspp-null and Dmp1-null mice showed a high level of biglycan in the expanded predentin [28, 32], indicating a potential role of biglycan in maintaining the predentin area in a non-mineralized status. A side-by-side comparison of the alizarin red stained 2.3Col 1 molar (Fig 7a, left panel) and the Gli1-tdTomato molar stained with biglycan polyclonal antibodies (Fig 7a, right panel) exhibited a close correlation of biglycan expression and a lack of mineral content in the predentin area. We also observed an expression pattern of nestin in the same Gli1-activated tdTomato molar. Nestin is mainly expressed in all odontoblast-processes in the predentin area (Fig $7 \mathrm{~b}$ ), although we do not know if a high level of nestin, like biglycan, inhibits mineralization in predentin at this stage.

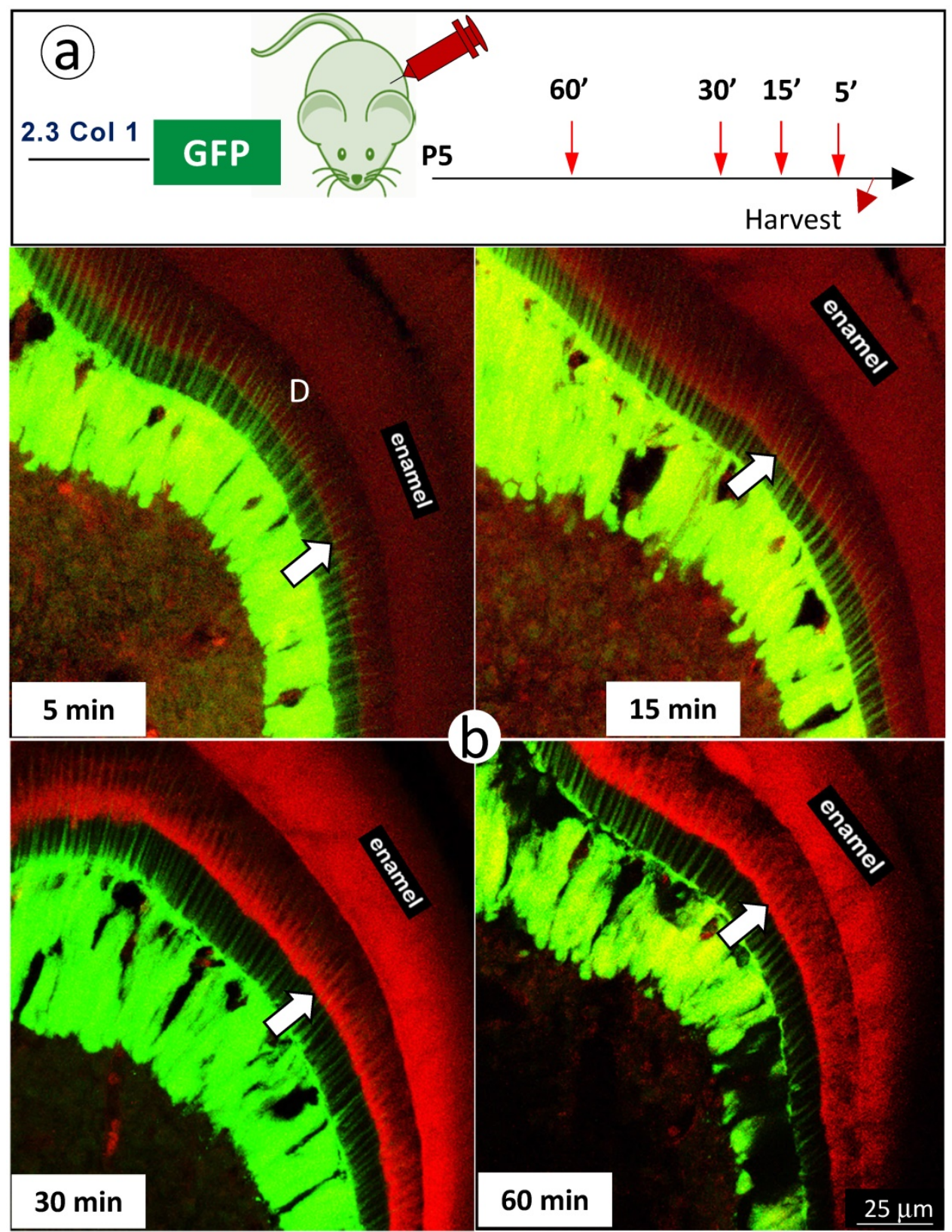

Figure 6. The short in vivo labeling minerals in dentin support the contribution of odontoblast processes to mineralization a. A schematic illustration of short time injections of Alizarin-red to 5-day-old 2.3 Col 1-GFP pups at 5-, 15-, 30- and 60-minute, respectively; and b. The distribution pattern of the red labeled mineral layer is more intense at the 15- and 60-minum time points (lower panels). It is worth noticing that the labeled mineral layers at the time of 15 -minute take place along with entire odontoblast processes; and that some of labels are observed in more broad dentin areas at the time of 60 minute. 


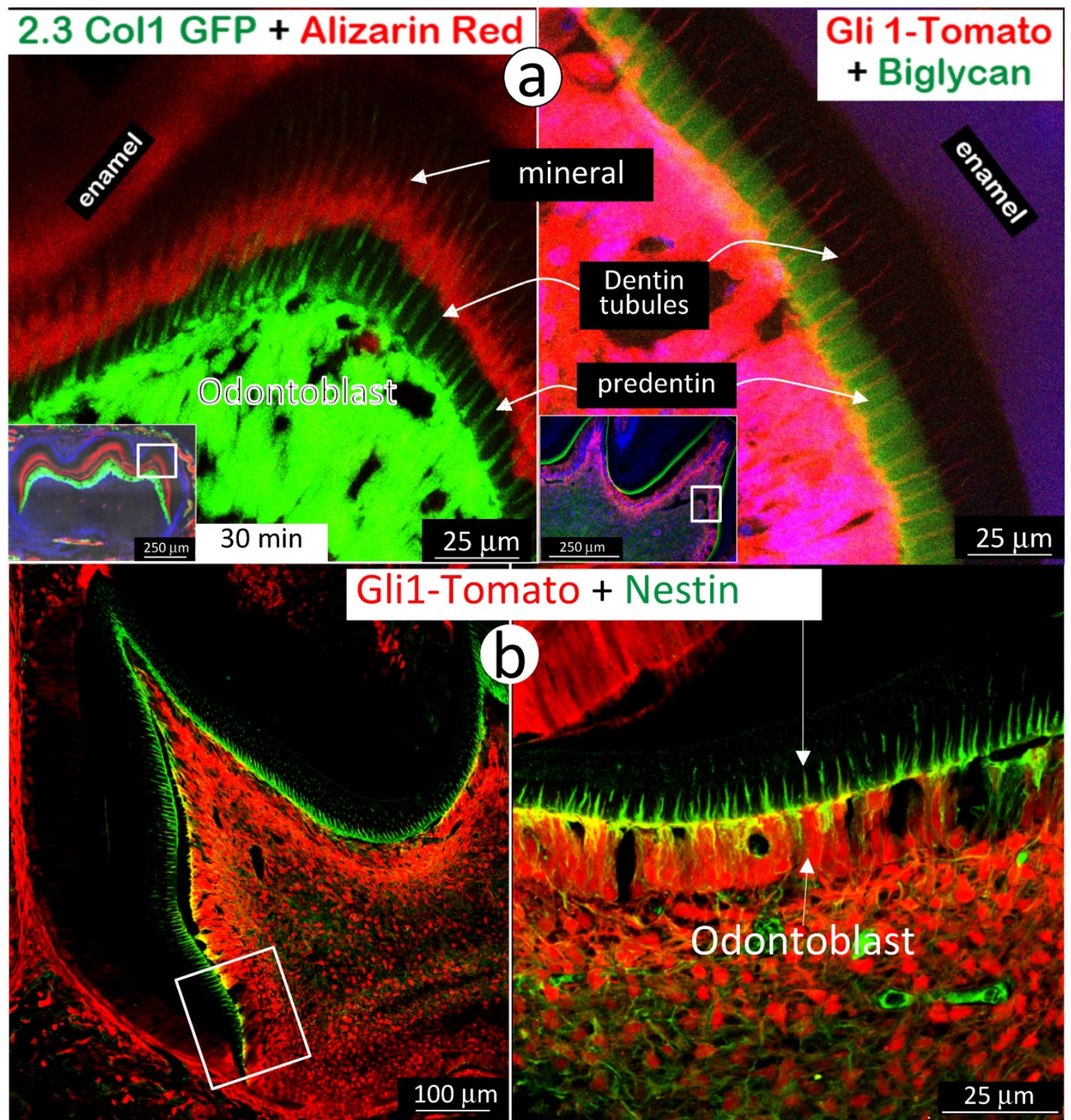

Figure 7. High expressions of biglycan and nestin in predentin but not in dentin. a. The 2.3 Col with alizarin-red label (reflecting the mineralization event; left panel) and the Glil-activated Td tomato (red color), in which the immuno-stained biglycan (green color) is restricted in the predentin (indicating an association with a lack of minerals in the predentin; right panel); and $\mathbf{b}$. The Glil-activated tomato molar slide, in which the immuno-stained nestin (green) is restricted in the odontoblast-process located in the predentin. Data indicate a likely role of these molecules in prevention of mineralization in predentin.

\section{Summary and Conclusion}

The mineralization-front theory is historically rooted in mineralization research fields for many decades. This theory is widely used to describe mineralization events in both osteogenesis and dentinogenesis. However, this model does not provide enough evidence to explain how minerals are evenly propagated from the pulp-end dentin to outer dentin. To address this issue, we modified the current research approaches by a) extending the mineral deposition windows of time from minutes to hours, instead of limiting the current mineralization assay on days and weeks only; b) switching a regular fluorescent microscope to a more powerful confocal microscope to reveal trace amount of minerals labelled along with dentin tubules; and c) using reporter mice, including the Gli1-CreERT2 activated tomato and the 2.3 Col1-GFP to mark odontoblast processes combined with mineral dye injections.

With a marriage of these technologies, we were able to clearly visualize the odontoblast-processes, full of numerous mini-branches, evenly spreading to entire dentin matrices with a high density of processes and a large diameter of the main process at the area adjacent to the tooth-pulp. We found that the minerals deposit along with entire odontoblast-processes and form many mineral collars surrounding odontoblast processes on both the pulp-end dentin and outer dentin. 
We therefore propose that it is the odontoblastprocess that directly contributes to mineralization, which is not only limited in the mineralization front at the edge of dentin and predentin.

Our finding also suggests that the so called "mineralization front line" is essentially composed of many individual mineral collars that is closely associated with dentin tubules, which are wide in diameter and rich in high density of the odontoblast processes at the edge of dentin and predentin (Supplementary Fig 6). We believe that these findings will shed new light on our understanding of dentin structure and function, as well as the mechanisms of mineralization.

\section{Supplementary Material}

Supplementary figures.

http://www.ijbs.com/v14p0693s1.pdf

\section{Acknowledgments}

We would like to thank Georgia K. Mitchell for editing the manuscript.

\section{Funding}

This study was supported in part by the NIH (R01DE025014 to J.Q.F), and National Natural Science Foundation of China (No. 81670962 to Z.W.).

\section{Author contributions}

C.L., Y.J., Y.R., K.W. performed experiments and analysis. C.L., H.Z., X.W., X.L., Z.W., J.F. prepared the manuscript. All authors were involved in interpretation of experiments and contributed to writing the paper.

\section{Competing Interests}

The authors have declared that no competing interest exists.

\section{References}

1. Frost HM, Roth H, Villanueva AR, Stanisavljevic S. Experimental multiband tetracycline measurement of lamellar osteoblastic activity. Henry Ford Hosp Med Bull. 1961;9:312-29.

2. Frost HM, Villaneuva AR. Tetracycline staining of newly forming bone and mineralizing cartilage in vivo. Stain Technol. 1960;35:135-8

3. Frost HM, Villanueva AR, Roth $\mathrm{H}$, Stanisavljevic $\mathrm{S}$. Tetracycline bone labeling. J New Drugs. 1961;1:206-16.

4. Goldberg M, Kulkarni AB, Young M, Boskey A. Dentin: structure, composition and mineralization. Front Biosci (Elite Ed). 2011:3:711-35.

5. Salomon JP, Lecolle S, Roche M, Septier D, Goldberg M. A radioautographic comparison of in vivo $3 \mathrm{H}$-proline and $3 \mathrm{H}$-serine incorporation in the pulpal dentine of rat molars: variations according to the different zones. J Biol Buccale. 1990;18(4):307-12.

6. Wu K, Frost HM. Bone formation in osteoporosis. Appositional rate measured by tetracycline labeling. Arch Pathol. 1969;88(5):508-10.

7. Frost HM, Vilanueva AR, Jett S, Eyring E. Tetracycline-based analysis of bone remodelling in osteopetrosis. Clin Orthop Relat Res. 1969;65:203-17.

8. Weinstock M, Leblond CP. Radioautographic visualization of the deposition of a phosphoprotein at the mineralization front in the dentin of the rat incisor. J Cell Biol. 1973;56(3):838-45.

9. Inage T, Toda Y. Phosphoprotein synthesis and secretion by odontoblasts in rat incisors as revealed by electron microscopic radioautography. Am J Anat. 1988;182(4):369-80.
10. Lundgren T, Linde A. Calcium ion transport kinetics during dentinogenesis: effects of disrupting odontoblast cellular transport systems. Bone Miner. 1992;19(1):31-44.

11. Dorvee JR, Gerkowicz L, Bahmanyar S, Deymier-Black A, Veis A. Chondroitin sulfate is involved in the hypercalcification of the organic matrix of bovine peritubular dentin. Arch Oral Biol. 2016;62:93-100.

12. Yoshiba K, Yoshiba N, Ejiri S, Iwaku M, Ozawa H. Odontoblast processes in human dentin revealed by fluorescence labeling and transmission electron microscopy. Histochem Cell Biol. 2002;118(3):205-12.

13. Dutra-Correa M, Anauate-Netto C, Arana-Chavez VE. Density and diameter of dentinal tubules in etched and non-etched bovine dentine examined by scanning electron microscopy. Arch Oral Biol. 2007;52(9):850-5.

14. Khatibi Shahidi M, Krivanek J, Kaukua N, Ernfors P, Hladik L, Kostal V, et al. Three-dimensional Imaging Reveals New Compartments and Structural Adaptations in Odontoblasts. J Dent Res. 2015;94(7):945-54.

15. Kalajzic Z, Liu P, Kalajzic I, Du Z, Braut A, Mina M, et al. Directing the expression of a green fluorescent protein transgene in differentiated osteoblasts: comparison between rat type I collagen and rat osteocalcin promoters. Bone. 2002;31(6):654-60.

16. Zhao H, Feng J, Ho TV, Grimes W, Urata M, Chai Y. The suture provides a niche for mesenchymal stem cells of craniofacial bones. Nat Cell Biol. 2015;17(4):386-96.

17. Feng JQ, Ward LM, Liu SG, Lu YB, Xie YX, Yuan BZ, et al. Loss of DMP1 causes rickets and osteomalacia and identifies a role for osteocytes in mineral metabolism. Nature Genetics. 2006;38(11):1310-5.

18. Sun Y, Chen L, Ma S, Zhou J, Zhang H, Feng JQ, et al. Roles of DMP1 Processing in Osteogenesis, Dentinogenesis and Chondrogenesis. Cells Tissues Organs. 2011;194(2-4):199-204.

19. Zhang H, Jiang Y, Qin CL, Liu Y, Ho SP, Feng JQ. Essential Role of Osterix for Tooth Root but not Crown Dentin Formation. Journal of Bone and Mineral Research. 2015;30(4):742-6.

20. Wang J, Muir AM, Ren Y, Massoudi D, Greenspan DS, Feng JQ. Essential Roles of Bone Morphogenetic Protein-1 and Mammalian Tolloid-like 1 in Postnatal Root Dentin Formation. J Endod. 2017;43(1):109-15.

21. Ren Y, Lin S, Jing Y, Dechow PC, Feng JQ. A novel way to statistically analyze morphologic changes in Dmp1-null osteocytes. Connect Tissue Res. 2014;55 Suppl 1:129-33.

22. Erben RG. Embedding of bone samples in methylmethacrylate: an improved method suitable for bone histomorphometry, histochemistry, and immunohistochemistry. J Histochem Cytochem. 1997;45(2):307-13.

23. Ma D, Zhang R, Sun Y, Rios HF, Haruyama N, Han X, et al. A novel role of periostin in postnatal tooth formation and mineralization. J Biol Chem. 2011;286(6):4302-9.

24. Miller SC, Omura TH, Smith LJ. Changes in dentin appositional rates during pregnancy and lactation in rats. J Dent Res. 1985;64(8):1062-4.

25. Martin DM, Hallsworth AS, Buckley T. A method for the study of internal spaces in hard tissue matrices by SEM, with special reference to dentine. J Microsc. 1978;112(3):345-52.

26. Feng JQ, Ward LM, Liu S, Lu Y, Xie Y, Yuan B, et al. Loss of DMP1 causes rickets and osteomalacia and identifies a role for osteocytes in mineral metabolism. Nat Genet. 2006;38(11):1310-5.

27. Keibl C, Fugl A, Zanoni G, Tangl S, Wolbank S, Redl H, et al. Human adipose derived stem cells reduce callus volume upon BMP-2 administration in bone regeneration. Injury-International Journal of the Care of the Injured. 2011;42(8):814-20.

28. Ye L, MacDougall M, Zhang S, Xie Y, Zhang J, Li Z, et al. Deletion of dentin matrix protein-1 leads to a partial failure of maturation of predentin into dentin, hypomineralization, and expanded cavities of pulp and root canal during postnatal tooth development. J Biol Chem. 2004;279(18):19141-8.

29. Lu Y, Ye L, Yu S, Zhang S, Xie Y, McKee MD, et al. Rescue of odontogenesis in Dmp1-deficient mice by targeted re-expression of DMP1 reveals roles for DMP1 in early odontogenesis and dentin apposition in vivo. Dev Biol. 2007;303(1):191-201.

30. Sasaki T, Garant PR. Structure and organization of odontoblasts. Anat Rec. 1996;245(2):235-49.

31. Nanci A, TenCate AR. Ten Cate's oral histology : development, structure, and function2018.

32. Sreenath T, Thyagarajan T, Hall B, Longenecker G, D'Souza R, Hong S, et al. Dentin sialophosphoprotein knockout mouse teeth display widened predentin zone and develop defective dentin mineralization similar to human dentinogenesis imperfecta type III. J Biol Chem. 2003;278(27):24874-80. 